\title{
3. Bogens und Woodwards Unterscheidung zwischen Daten und Phänomenen
}

3.1 Dreigliedrige Wissenschaftsmodelle und die Daten-Phänomen-
Unterscheidung

James Bogen und James Woodward versuchen in einer Reihe von Aufsätzen aufzuzeigen, dass zweigliedrige Wissenschaftsmodelle keine angemessene Beschreibung der wissenschaftlichen Praxis erlauben. ${ }^{8}$ Für eine solche müsse vielmehr eine Triade von Daten, Phänomenen und Theorien in Betracht gezogen werden, da wissenschaftliche Theorien in der Regel andere Sachverhalte erklären und vorhersagen als die, die wir mit bloßem Auge beobachten. Erstere bezeichnen Bogen und Woodward als Phänomene, letztere als Daten. In diesem Kapitel sollen erste Schritte zur Klärung dieser Unterscheidung unternommen werden.

Eine erste Idee, worin die Unterscheidung besteht, vermitteln die zahlreichen Beispiele, die Bogen und Woodward zur Illustration anführen. Als Beispiele für Daten nennen sie Nebelkammeraufnahmen, bestimmte Entladungsmuster in Teilchendetektoren oder die Zeigerstände eines Thermometers. Phänomene, für die diese Daten (unter günstigen Umständen) Belege liefern, sind schwache neutrale Ströme, der Zerfall des Protons oder der Schmelzpunkt von Blei. ${ }^{9}$ Insbesondere das letztgenannte Phänomen, der Schmelzpunkt von Blei, ist eines von Bogens und Woodwards Paradebeispielen, um die Unzulänglichkeit zweigliedriger Wissenschaftsmodelle aufzuzeigen. Dies ist überraschend, denn auf den ersten Blick erscheint es so, als sei der Schmelzpunkt von Blei beobachtbar, also ein Phänomen im Sinne der traditionellen Phänomenauffassung. Man muss, so hat es den Anschein, bloß Blei zum Schmelzen bringen und dabei die entsprechende Temperatur mit einem Thermometer messen, dann kann man (mit Hilfe des Thermometers) beobachten, dass Blei bei $327,46^{\circ} \mathrm{C}$ schmilzt. Bogen und Woodward wenden jedoch ein, dass dies keineswegs das sei, was Wis-

\footnotetext{
${ }^{8}$ Vgl. Bogen und Woodward (1988; 1992; 2003), Bogen (2009a; 2009b) und Woodward $(1989 ; 2000 ; 2009)$.

${ }^{9}$ Vgl. Bogen und Woodward (1988), S. 306-308. In ihren zahlreichen Artikeln geben Bogen und Woodward darüber hinaus etliche weitere Beispiele, auch aus anderen wissenschaftlichen Disziplinen, beispielsweise aus der Psychologie.
} 
senschaftler tatsächlich tun, wenn sie den Schmelzpunkt von Blei bestimmen. Der Schmelzpunkt von Blei wird nicht mittels einmaligen Ablesens eines Thermometers an einer Versuchsapparatur bestimmt, sondern muss mit Hilfe einer Vielzahl von Messungen bestimmt werden. Auch wenn die Messapparatur gut funktioniert und systematische Fehler ausgeschlossen werden können, wird man dabei feststellen, dass sich die einzelnen Messergebnisse, die Daten, (leicht) voneinander unterscheiden. Zur Erklärung dieses Umstandes wird angenommen, dass die Daten sowohl durch den „wahren“ Schmelzpunkt als auch durch zahlreiche andere kleine und „zufällige" Ursachen zustande kommen, die nicht kontrolliert werden können und die zumeist auch unbekannt bleiben. Kann man im Hinblick auf diese „zufälligen Fehler“ Aussagen derart machen, dass sie voneinander unabhängig sind (Unkorreliertheit), dass sie die gleiche Varianz haben (Homoskedastizität) und dass sie genauso wahrscheinlich zu einer Erhöhung wie zu einer Erniedrigung des Wertes führen (Erwartungswert von Null), dann kann man davon ausgehen, dass die Messergebnisse normalverteilt sind und dass der Mittelwert der Verteilung eine gute Abschätzung für den „wahren Wert“ abgibt. Das Phänomen, dass der Schmelzpunkt von Blei bei $327,46^{\circ} \mathrm{C}$ liegt, beobachten wir somit nicht, sondern wir erschließen es auf Grundlage vorliegender Daten und spezifischer Voraussetzungen, wie mathematisch-statistischer Methoden. Erst der auf diese Weise erschlossene Wert ist es dann, der als Beleg für oder gegen die Geltung bestimmter Theorien herangezogen werden kann bzw. der durch bestimmte physikalische Theorien, z.B. durch Theorien über die atomaren Bindungen in Festkörpern, erklärt wird.

Ausgehend von diesen und weiteren Beispielen identifizieren Bogen und Woodward die folgenden Charakteristika von Daten und Phänomenen. $D a$ ten seien Messergebnisse, die dem menschlichen Wahrnehmungssystem zugänglich seien und die man einer öffentlichen Prüfung unterziehen könne. Daten seien an bestimmte experimentelle Kontexte, insbesondere an bestimmte Messapparaturen, gebunden und könnten nicht außerhalb dieser Kontexte auftreten. Sie würden in der Regel von so verschiedenen und zahlreichen Ursachen hervorgebracht, dass es praktisch nicht möglich sei, eine Theorie $\mathrm{zu}$ konstruieren, die ihr Zustandekommen vorhersage oder angebe, wie die verschiedenen Ursachen zusammenwirken, um diese Daten hervorzubringen. Aufgrund der Gebundenheit an spezifische Messkontexte und der kausalen Abhängigkeit von einer Vielzahl von Ursachen be- 
zeichnen Bogen und Woodward Daten als „idiosynkratisch“. ${ }^{10}$ Darüber hinaus - das ist ein entscheidender Punkt - sei es gar nicht erforderlich, die Daten auf diese Weise zu erklären, um erfolgreich Wissenschaft zu betreiben. Vielmehr sei das Gegenteil der Fall: Nur Absehung von den einzelnen Datenpunkten und die theoretische Behandlung stabiler und regulärer Phänomene ermögliche die Allgemeinheit, die wissenschaftliche Erkenntnisse so nützlich mache. ${ }^{11}$

Phänomene seien hingegen nicht in der beschriebenen Weise idiosynkratisch. Sie träten in unterschiedlichen Kontexten auf und seien in der Regel nicht an spezifische experimentelle Methoden gebunden, sondern könnten mittels unterschiedlicher Verfahren nachgewiesen werden (wobei diese unterschiedlichen Verfahren natürlich verschiedene Arten von Daten hervorbringen). Phänomene seien zudem im Gegensatz zu Daten Gegenstand wissenschaftlicher Erklärungen und Vorhersagen. Die Phänomene, die wissenschaftliche Theorien erklären und vorhersagen, seien dabei in aller Regel nicht die Sachverhalte, die wir beobachten. Dies wiederum liege nicht nur daran, dass viele wissenschaftliche Theorien von Entitäten handeln, die viel zu klein oder zu weit entfernt sind, um sie beobachten zu können, sondern auch Phänomene, die beobachtbare Entitäten wie Bleistücke betreffen, würden in der Regel nicht beobachtet. Aufgrund dieser Unterschiede seien dreigliedrige Wissenschaftsmodelle, mit der in Abbildung 2 illustrierten Struktur, der wissenschaftlichen Praxis gegenüber angemessener als zweigliedrige.

\footnotetext{
${ }^{10}$ Bogen und Woodward (1988), S. 317.

11 „The fact that theories are better at predicting and explaining phenomena than data isn't such a bad thing. For many purposes, theories that predict and explain phenomena would be more illuminating and more useful for practical purposes than theories (if there were any) that predicted or explained members of a data set. [...] For most purposes, the former theory would be preferable to the latter at the very least because it applies to so many more cases." Bogen (2009b), Abschnitt 10. Vgl. auch Bogen und Woodward (1988), S. 326.
} 


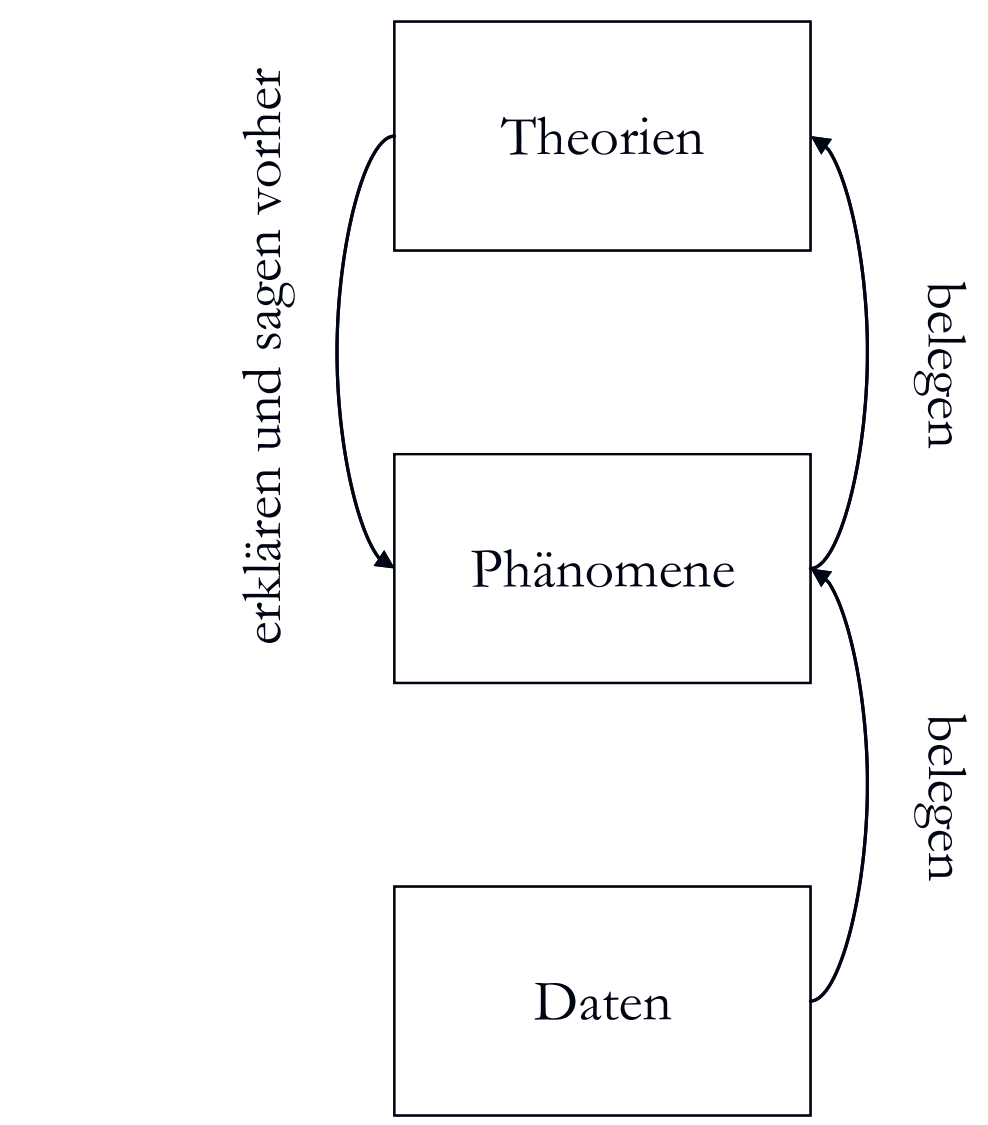

Abbildung 2: Dreigliedriges Wissenschaftsmodell nach Bogen und Woodward

Darüber hinaus sprechen Bogen und Woodward davon, dass die Phänomene „relativ stabile und allgemeine Merkmale der Welt“ seien. ${ }^{12}$ Mit dieser Formulierung legen sich Bogen und Woodward auf die Position des Wissenschaftlichen Realismus fest. ${ }^{13}$ In der hier vorliegenden Arbeit soll es jedoch gerade um die erkenntnistheoretische Bewertung der DatenPhänomen-Unterscheidung gehen, also darum, festzustellen, ob sich unter Rückgriff auf diese Unterscheidung für den Wissenschaftlichen Realismus oder Antirealismus argumentieren lässt. Deshalb soll es im Rahmen der hier zu leistenden Begriffsklärung offen bleiben, ob die Phänomene im Sinne des Realismus als von uns unabhängige Bestandteile der Welt oder im Sinne eines Antirealismus beispielsweise als nützliche, aber von uns erschaffene Konstrukte verstanden werden müssen. Mithin soll dieses letzte Merkmal, das Bogen und Woodward anführen, bei meiner Betrachtung nicht vorausgesetzt werden.

\footnotetext{
12 "Phenomena [...] are relatively stable and general features of the world which are potential objects of explanation and prediction by general theory." Woodward (1989), S. 393.

13 Auch an anderen Stellen bekennen sich Bogen und Woodward ausdrücklich zum Realismus. Vgl. z.B. Bogen und Woodward (1988), S. 337.
} 
Aber auch abgesehen von der Frage, ob wissenschaftliche Phänomene realistisch interpretiert werden sollten, wird aus den bisherigen Überlegungen nicht klar, worin genau die wesentlichen Unterschiede zwischen Daten und Phänomenen bestehen. Handelt es sich bei wissenschaftlichen Phänomenen beispielsweise um prinzipiell unbeobachtbare Entitäten? Sind Daten und Phänomene immer unterschiedliche Entitäten oder kann etwas Datum und Phänomen zugleich sein? Wie wird ein Phänomen von einem anderen abgegrenzt? ${ }^{14}$ Wie genau ist das Verhältnis von Phänomenen und Theorien zu spezifizieren? Kurz: Was zu einem adäquaten Verständnis von Bogens und Woodwards Unterscheidung fehlt, ist eine Angabe der begrifflichen Merkmale des Daten- und des Phänomenbegriffs. Bogen und Woodward unterbreiten hierfür kein Angebot. Sie arbeiten mit zahlreichen Illustrationen und Beispielen, verwenden sehr oft Formulierungen wie ,in der Regel sind Phänomene so und so beschaffen“ oder ,häufig haben Phänomene diese und jene Eigenschaften" und verlassen sich augenscheinlich darauf, dass hierdurch hinreichend klar wird, worin die Daten-PhänomenUnterscheidung besteht. ${ }^{15}$ Ich werde im weiteren Verlauf versuchen, diese Lücke zu schließen und eine adäquate Explikation der Unterscheidung zu entwickeln. Dabei wird man sehen, dass sich die Unterscheidung keineswegs als so klar und intuitiv einleuchtend erweist, wie es auf den ersten Blick erscheinen mag, und dass zahlreiche Autoren, die auf die Unterscheidung Bezug nehmen, sie meines Erachtens missverstehen.

\subsection{Phänomene im wissenschaftlichen Sprachgebrauch}

Den Ausgangspunkt für die weitere Interpretation der Daten-PhänomenUnterscheidung bildet der wissenschaftliche Sprachgebrauch. Wissenschaftler verwenden die Ausdrücke „Datum“ und „Phänomen“ regelmäßig und meines Erachtens sollte die Daten-Phänomen-Unterscheidung auf eine Weise gefasst werden, die wesentlichen Aspekten des wissenschaftlichen Sprachgebrauchs gerecht wird. Warum ich dieser Auffassung bin, werde ich im folgenden Abschnitt 3.3 begründen. Zuvor sollen wichtige Merkmale beider Begriffe herausgearbeitet werden.

Auf den Datenbegriff müssen in diesem Zusammenhang nicht viele Worte verwendet werden. Er hat sowohl bei Bogen und Woodward als auch im

\footnotetext{
${ }^{14}$ Machamer (2009), S. 2 wirft dieses Problem anhand der Frage auf, ob es sich es sich bei Schizophrenie um ein psychologisches Phänomen oder um mehrere handelt.

${ }^{15}$ Woodward (2009), S. 7 räumt ein, dass dies eine Schwäche des Ansatzes ist.
} 
wissenschaftlichen Sprachgebrauch eine klar umrissene Bedeutung: Etwas ist genau dann ein Datum, wenn es beobachtet wurde und als Beleg für wissenschaftliche Aussagen herangezogen werden kann. Die Bedeutung des Phänomenbegriffs zu klären, erweist sich hingegen als schwieriger. Dem entsprechenden sprachlichen Ausdruck „Phänomen“ begegnet man in verschiedenen Kontexten und wenn man klären möchte, was er bedeutet, dann muss man sich ansehen, in welchen Zusammenhängen und auf welche Weise der Ausdruck verwendet wird. Ich beschränke mich hier auf die Verwendung des Ausdrucks in naturwissenschaftlichen Kontexten. ${ }^{16}$ Beispiele für diese entnehme ich naturwissenschaftlichen Fachpublikationen. Anhand von Beispielen aus einer aktuellen Ausgabe der Zeitschrift Nature möchte ich zwei wesentliche Merkmale des Phänomenbegriffs vorstellen:

"In the phenomenon known as the Aharonov-Bohm effect, magnetic forces seem to act on charged particles such as electrons - even though the particles do not cross any magnetic field lines. Is this evidence for electromagnetic forces that work in new and unsuspected ways?"17

"The presence of a liquid-gas transition was noted to be very remarkable because there are few, if any, other experimentally known instances in localized spin systems. [...] No mechanism was known to account for this phenomenon, and our theory of magnetic monopoles fills this gap."18

Diese Aussagen stehen exemplarisch für die Art und Weise, wie Wissenschaftler über Phänomene sprechen. Dass diese Aussagen typisch sind, ist natürlich eine empirische Hypothese. Aber ich denke, es ist leicht, sich von ihrer Wahrheit zu überzeugen, beispielsweise indem man die Onlinesuche naturwissenschaftlicher Fachzeitschriften benutzt und nach dem Stichwort „Phänomen“" bzw. „phenomenon“ sucht. Bei Nature erhält man auf diese

\footnotetext{
${ }^{16}$ Auch in der Umgangssprache sprechen wir von Phänomenen. („Gerd Müller ist ein Phänomen. Er hat 365 Tore in 427 Bundesligaspielen erzielt.") Und auch in der Philosophie wird der Begriff häufig, wenngleich nicht einheitlich, verwendet. Einen Überblick über den philosophischen Phänomenbegriff liefert der entsprechende Eintrag im Historischen Wörterbuch der Philosophie: Vgl. Ritter and Gründer (1989), Bd. 7, S. 461-471. Gemeinsamkeiten und Unterschiede zwischen der Begriffsverwendung in Alltags-, philosophischen und naturwissenschaftlichen Kontexten thematisiere ich nicht weiter.

17 Tonomura und Nori (2008), S. 298.

${ }^{18}$ Castelnovo, Moessner, und Sondhi (2008), S. 44.
} 
Weise eine fünfstellige Trefferzahl. ${ }^{19}$ Liest man diese Ergebnisse quer, wird schnell deutlich, dass obige Beispiele repräsentativ sind.

Wenn es sich bei diesen Aussagen tatsächlich um exemplarische Aussagen über Phänomene handelt, dann ist es möglich, zentrale Merkmale des Phänomenbegriffs aus diesen Aussagen zu extrahieren. Diese zentralen begrifflichen Merkmale sind die funktionalen Rollen, die Phänomene in der wissenschaftlichen Praxis spielen:

i) Phänomene sind potentielle Belege für Theorien (erstes Zitat) und

ii) Phänomene sind potentielle Explananda von Theorien (zweites $\mathrm{Zi}$ tat). ${ }^{20}$

Später werde ich diese beiden funktionalen Rollen noch genauer erläutern, indem ich diskutiere, was Belege und Erklärungen sind. ${ }^{21}$ An dieser Stelle reicht jedoch ein vortheoretisches Verständnis des Beleg- und Erklärungsbegriffs aus, um hinreichend gut zu verstehen, was mit i) und ii) ausgesagt wird. Allerdings sind an diesem Punkt zwei andere Erläuterungen erforderlich, die die Qualifikation ,potentiell“ betreffen, die ich in den Formulierungen von i) und ii) benutzt habe.

Erstens stellt sich die Frage, warum ich davon spreche, dass Phänomene potentielle Belege und Explananda sind und nicht davon, dass sie Belege und Explananda sind. Der Grund hierfür ist simpel: Es gibt zahlreiche Fälle, in denen Wissenschaftler auf ein bestimmtes Phänomen stoßen, aber weder über eine Theorie verfügen, die durch das Phänomen erklärt wird, noch über eine, die durch das Phänomen bestätigt wird. Man denke an

${ }^{19}$ Die Größenordnung der Treffer liegt im gleichen Bereich wie die Treffer bei der Suche nach anderen allgemeinen wissenschaftlichen Ausdrücken wie z.B. „Erklärung“, „Modell“ oder „Theorie“. Dies spricht dafür, dass „Phänomen“ genau wie diese Ausdrücke eine gängige Vokabel des wissenschaftlichen Sprachgebrauchs ist. Deshalb ist der entsprechende Begriff prima facie ebenso untersuchenswert wie die anderen Begriffe.

${ }^{20}$ Meine Rede von funktionalen Rollen ist nicht $\mathrm{zu}$ verwechseln mit der Rede von funktionalen Rollen im Rahmen einer funktionalistischen Theorie geistiger Zustände, wie sie beispielsweise David Lewis (1966) in der Philosophie des Geistes vertritt. Funktionale Rollen im Sinne des Funktionalismus sind kausale Rollen, die geistige Zustände, wie das Schmerzen-Haben, innehaben. Funktionale Rollen, so wie ich den Begriff verwende, bezeichnen hingegen keine kausalen Rollen, sondern Funktionen, die bestimmte Sachverhalte in der wissenschaftlichen Praxis innehaben. Diese Funktionen sind ihre funktionalen Rollen.

${ }^{21} \mathrm{Vgl}$. Abschnitt 5.2. 
Becquerels Entdeckung der radioaktiven Strahlung. Das Phänomen, dass Uran Photoplatten schwärzt, entdeckte Becquerel zufällig und man verfügte zunächst über keine Theorie, die das Phänomen hätte erklären können. Dennoch würden wir sagen, dass Becquerel ein bis dahin unbekanntes Phänomen entdeckt hat, gerade weil Becquerel seine Entdeckung als potentielles Explanandum behandelte und nach einer entsprechenden Erklärung suchte. Wir benutzen den Phänomenbegriff bereits dann, wenn wir noch auf der Suche nach einer adäquaten Erklärung sind und nicht erst dann, wenn wir über eine solche verfügen.

Zweitens muss genauer erläutert werden, in welcher Weise der Ausdruck „potentiell“ verstanden werden sollte. Fasste man diese Qualifikation im Sinne eines logischen, metaphysischen oder naturgesetzlichen Möglichkeitsoperators auf, so wäre das nicht stark genug. Denn es ist selbstverständlich im Hinblick auf jeden Sachverhalt, mit dem wir in wissenschaftlichen Zusammenhängen konfrontiert werden, logisch, metaphysisch und naturgesetzlich möglich, dass er als Beleg oder als Explanandum dienen kann. Ein solches Verständnis würde die beiden Begriffsmerkmale trivialisieren. Wir bezeichnen in naturwissenschaftlichen Forschungskontexten nicht jeden beliebigen Sachverhalt als Phänomen, sondern wir wenden den Begriff nur auf solche an, die wir im Sinne der beiden funktionalen Rollen behandeln. Das heißt so viel wie, dass wir entweder de facto über eine Theorie verfügen, mit der wir ein Phänomen erklären, oder dass wir zumindest versuchen, eine solche Theorie zu finden. (Analoges gilt für die Belegrolle.) Man könnte sagen, dass der Ausdruck Phänomen eine Art „Ehrentitel“" ist, den Wissenschaftler bestimmten Sachverhalten zusprechen (etwa in der folgenden Weise: „Von den unendlich vielen Sachverhalten, die der Fall sind, interessiere ich mich für das Phänomen, dass Uran Photoplatten schwärzt."). Dies ist der Sinn, in dem die Qualifikation ,potentiell“ in i) und ii) verstanden werden sollte.

\subsection{Ist die Daten-Phänomen-Unterscheidung deskriptiv oder stipulativ?}

An dieser Stelle steht immer noch die Frage im Raum, warum der wissenschaftliche Sprachgebrauch überhaupt dazu dienlich sein sollte, zu klären, wie eine philosophische Unterscheidung wie die Daten-PhänomenUnterscheidung verstanden werden muss. Warum sollte die Bedeutung des Ausdrucks „Phänomen“ im wissenschaftlichen Sprachgebrauch die gleiche 
sein, wie die des gleichlautenden Ausdrucks im Rahmen der philosophischen Überlegungen von Bogen und Woodward? In Kapitel 2 habe ich die traditionelle Auffassung wissenschaftlicher Phänomene vorgestellt und dabei deutlich gemacht, dass deren Phänomenbegriff nicht darauf ausgelegt ist, dem gegenwärtigen wissenschaftlichen Sprachgebrauch gerecht $\mathrm{zu}$ werden. Der Ausdruck ist dort ausdrücklich für beobachtbare Sachverhalte reserviert, auch wenn Wissenschaftler selbst unbeobachtbare Sachverhalte wie den Aharonov-Bohm-Effekt als Phänomene bezeichnen. Man hat es dort mit einer, wie ich es im Folgenden nennen werde, stipulativen Verwendung des Ausdrucks zu tun. ${ }^{22}$ Als stipulativ bezeichne ich die Art und Weise einen sprachlichen Ausdruck zu verwenden, wenn der Ausdruck als terminus technicus in einer Theorie eingeführt wird, die Bedeutung des Ausdrucks also speziell für diese Theorie definiert wird. Dabei werden zwar häufig sprachliche Ausdrücke verwendet, die wir auch in der Umgangssprache oder wie im hier betrachteten Fall im naturwissenschaftlichen Sprachgebrauch benutzen, aber es wird nicht versucht, die theoretische mit der umgangssprachlichen bzw. naturwissenschaftlichen Verwendungsweise zur Deckung zu bringen. Im Gegensatz dazu werden andere Ausdrücke in (philosophischen) Theorien deskriptiv verwendet. Dabei wird das Ziel verfolgt, die umgangssprachliche bzw. naturwissenschaftliche Verwendungsweise eines Ausdrucks weitestgehend einzufangen und zu erhellen. Der Wissensbegriff in der Erkenntnistheorie ist ein Beispiel hierfür. Im Hinblick auf Bogens und Woodwards Unterscheidung stellt sich nun die Frage, ob sie als deskriptiver Vorschlag zur Erhellung des Phänomenbegriffs (und auch des Datenbegriffs) zu verstehen ist. In diesem Abschnitt möchte ich plausibel machen, dass dies der Fall ist.

Ausgangspunkt meiner Überlegung ist die Beobachtung, dass sich die Art und Weise, wie Bogen und Woodward Phänomene charakterisieren, er-

\footnotetext{
${ }^{22}$ Man beachte, dass die Dichotomie zwischen "deskriptiv“ und „stipulativ“ eine andere ist als die zwischen deskriptiven und normativen Ansätzen, die für wissenschaftstheoretische Betrachtungen ebenfalls eine Rolle spielt. Im Sinne der letztgenannten Unterscheidung versuchen deskriptive Ansätze zu beschreiben, wie die Wissenschaft faktisch vorgeht, während normative Ansätze angeben, wie die Wissenschaft vorgehen sollte. Bei der Unterscheidung zwischen deskriptiven und stipulativen Ansätzen geht es hingegen darum, wie Begrifflichkeiten innerhalb des philosophischen Diskurses verwendet werden. Deskriptive Ansätze versuchen die Begriffe so zu benutzen und auszuarbeiten, dass sie den Begriffen, die im wissenschaftlichen Objektdiskurs benutzt werden, so weit wie möglich entsprechen. Stipulative Ansätze hingegen benutzten ggf. gleich lautende sprachliche Ausdrücke, stipulieren aber deren Bedeutung im Rahmen der jeweiligen philosophischen Theorie.
} 
staunlich gut mit dem wissenschaftlichen Sprachgebrauch deckt. Auf dieser Basis ist ein erster Grund, warum die Unterscheidung als deskriptive Unterscheidung verstanden werden sollte, dass Bogen und Woodward den Ausdruck „Phänomen“ an keiner Stelle anhand einer expliziten Definition einführen. Dies würde man aber erwarten, wenn sie eine spezifische Bedeutung für den Ausdruck stipulieren wollten. Vielmehr benennen sie Beispiele aus der wissenschaftlichen Praxis, an denen sie den Unterschied zwischen Daten und Phänomenen aufzeigen und anhand derer sie die Charakteristika von Phänomenen erarbeiten. Der zweite und entscheidende Grund, warum ich der Auffassung bin, dass man die Unterscheidung als deskriptive Unterscheidung verstehen sollte, ist jedoch, dass dieses Verständnis zu generellen Entwicklungen innerhalb der Wissenschaftstheorie passt. Heutzutage sind deskriptive Ansätze in der Wissenschaftstheorie populär und gelten als erfolgreicher als stipulative Ansätze. Man denke beispielsweise an die Debatte über die Natur wissenschaftlicher Theorien. Um zu klären, was wesentliche Charakteristika einer wissenschaftlichen Theorie sind, gehen Philosophen so vor, dass sie sich ansehen, welche Arten von Entitäten als Theorien bezeichnet werden, und versuchen, gemeinsame Charakteristika all dieser Entitäten zu identifizieren. Innerhalb dieser Debatte wird es als schwerwiegendes Manko des sog. syntaktischen Ansatzes angesehen, dass er sich auf viele Entitäten nicht anwenden lässt, die Wissenschaftler als Theorien bezeichnen, wie z.B. die Darwin'sche Evolutionstheorie oder Theorien über das Aussterben der Dinosaurier. Ähnliche Beispiele lassen sich in vielen anderen wissenschaftstheoretischen Debatten finden. Wissenschaftstheoretiker versuchen Aufschluss darüber zu erhalten, was Wissenschaft ist, was die wissenschaftliche Methodologie auszeichnet und welche Art von Erkenntnissen uns die Wissenschaft vermittelt. Um dieses Ziel zu erreichen, analysieren Wissenschaftstheoretiker zum einen Begriffe, die innerhalb wissenschaftlicher Theorien eine Rolle spielen (wie den Feldbegriff oder den Atombegriff), und zum anderen Begriffe, mit denen wir über die Wissenschaft sprechen (wie den Erklärungsbegriff, den Theorienbegriff oder eben den Phänomenbegriff). ${ }^{23}$

${ }^{23}$ Auch für Peter Achinstein sind allgemeine Begriffe, mit denen wir über Wissenschaft sprechen, der primäre Forschungsgegenstand der Wissenschaftstheorie. So beschreibt er sowohl das Anliegen der Wissenschaftstheorie im Allgemeinen als auch das seines mittlerweile klassischen Werkes Concepts of Science im Speziellen folgendermaßen: „,This book is concerned with concepts important for understanding the nature of science. Those to be discussed are not themselves scientific concepts, they are not one scientists usually examine in the course of their work, nor are they concepts 
Die Wissenschaftstheorie zielt letztlich auf die epistemische Beurteilung unserer wissenschaftlichen Praxis ab. Um eine solche Beurteilung leisten zu können, bedarf es Klarheit darüber, worin die Natur der zentralen Aspekte der wissenschaftlichen Praxis (d.h. z.B. Theorien, Erklärungen oder eben auch Phänomene) besteht und es besteht darüber hinaus weitestgehend Einigkeit darüber, dass man, um solche Klarheit zu erlangen, prüfen muss, welche Art von Entitäten wir mit den entsprechenden sprachlichen Ausdrücken bezeichnen und welche gemeinsamen Merkmale diese Entitäten verbinden. Wenn man zugesteht, dass ein solches Vorgehen die erfolgversprechendste philosophische Methodologie ist, die uns im Rahmen der Wissenschaftstheorie zur Verfügung steht, dann ist es nahe liegend, auch Bogen und Woodward als Vertreter eines solchen Ansatzes aufzufassen.

\subsection{Phänomene als Sachverhalte}

Ich habe im Vorhergehenden bereits an verschiedenen Stellen davon gesprochen, dass Phänomene Sachverhalte sind. Und auch im Folgenden werde ich immer wieder von Phänomenen als Sachverhalten sprechen. Dies wirft drei Fragen auf: Was genau sind Sachverhalte? Wie komme ich zu der Behauptung, Phänomene seien Sachverhalte? Hat die Zuordnung wissenschaftlicher Phänomene zur Klasse der Sachverhalte philosophische Konsequenzen?

Beginnen wir mit der ersten Frage. Sachverhalte sind Entitäten, auf die wir uns in dass-Sätzen oder substantivierten Infinitivkonstruktionen beziehen. So ist es zum Beispiel ein Sachverhalt, dass Peter $80 \mathrm{~kg}$ wiegt. Sachverhalte haben eine komplexe Struktur, sie „bestehen“ in gewissem Sinn aus Gegenständen und ihren Eigenschaften sowie Relationen zwischen Gegenständen. Sie sind, so kann man etwas ungelenk sagen, „Verbindungen von Gegenständen und Eigenschaften“. ${ }^{24}$ Die Klasse der Sachverhalte zerfällt

appropriate for one science but not another. Rather, they are very general ones applicable to all sciences, and would be invoked in talking about, rather than within, these sciences. They are the province of the philosopher of science." Achinstein (1968), S. vii.

${ }^{24}$ Vgl. Schantz (1996) zu einer ausführlichen Sachverhaltstheorie. Wichtig ist in diesem Zusammenhang, dass Sachverhalte eine innere Ordnung haben. Der Sachverhalt, dass Peter Anna liebt, ist ein anderer als der, dass Anna Peter liebt, obwohl die gleichen Personen und die gleiche relationale Eigenschaft in beiden Sachverhalten vorkommt. 
wiederum in die Teilklasse der bloß möglichen Sachverhalte und die der Sachverhalte, die der Fall sind. Letztere bezeichnet man als Tatsachen.

Dass Phänomene zur ontologischen Kategorie der Sachverhalte gehören, ergibt sich, anders als ihre funktionalen Rollen, nicht eindeutig aus der Verwendungsweise des sprachlichen Ausdrucks. Wissenschaftler sagen nicht nur Sätze wie „Ich erforsche das Phänomen, dass Licht in Gravitationsfeldern abgelenkt wird“ sondern auch Sätze wie „Das Phänomen, das ich erklären möchte, ist der Prozess der Sternentstehung“ oder „Das Phänomen, das mich interessiert, ist die Sonnenfinsternis vom 3. Mai 1715“. Offensichtlich werden also unter anderem auch Prozesse und Ereignisse als Phänomene bezeichnet. Bogen und Woodward, deren Position später ausführlich diskutiert werden soll, schreiben deshalb über Fragen der ontologischen Klassifizierung von Phänomenen Folgendes:

„Phenomena seem to fall into many different traditional ontological catego-
ries - they include particular objects, objects with features, events, processes,
and states. Perhaps some phenomena are best thought of as having a structure
more like that traditionally ascribed to facts or states of affairs. [...]For our pur-
poses, what matters most about phenomena is the distinctive role they play in
connection with explanation and prediction [...] For our purposes, anything
which can play this role [...] can qualify as a phenomenon, and this is why (like
the scientists whose activity we claim to be describing) we are inclined to be
somewhat casual about matters of ontological classification." 25

Ich halte Bogens und Woodwards Beobachtung für korrekt. Die Sprachverwendung lässt keine eindeutige ontologische Klassifikation von Phänomenen zu und ein ,zwangloser“ Umgang mit ontologischen Fragen scheint angezeigt. In diesem Sinne ist meine Zuordnung von Phänomenen zur Klasse der Sachverhalte bloß pragmatisch begründet. Ich halte diese Zuordnung für nützlich und zwar aus folgenden Gründen: Sie deckt sich mit der Charakterisierung von Phänomenen über ihre funktionalen Rollen, denn es besteht (im Rahmen philosophischer Theorien, wahrscheinlich aber nicht im alltäglichen Sprachgebrauch) Einigkeit darüber, dass Sachverhalte die Relata sowohl der Erklärungs- als auch der Belegrelation sind. Wir fragen nach einer Erklärung für den Sachverhalt, dass der Himmel blau ist, und der Sachverhalt, dass im Doppelspaltversuch ein Interferenzmuster entsteht, ist ein Beleg dafür, dass Licht Welleneigenschaften hat. Zweitens ist es zumindest eine übliche Verwendungsweise des Ausdrucks

${ }^{25}$ Bogen und Woodward (1988), S. 321-322. 
„Phänomen“, ihn zur Bezeichnung von Sachverhalten zu benutzen. Die Phrase „das Phänomen, dass..." findet man regelmäßig im wissenschaftlichen Sprachgebrauch. Zudem interessiert man sich in naturwissenschaftlichen Kontexten oft für allgemeine Regularitäten. Wenn beispielsweise davon gesprochen wird, dass es eine Aufgabe der Astrodynamik sei, die Bewegungen der Himmelskörper zu erklären, so werden nicht nur einzelne raum-zeitliche Ereignisse erklärt, sondern auch allgemeine Tatsachen, wie die, dass, unter Voraussetzung bestimmter Anfangs- und Randbedingungen sowie der Gesetze der Mechanik, die Planeten auf Ellipsenbahnen um die Sonne kreisen. Darüber hinaus werden in der Physik häufig die Ausdrücke „Effekt" und „Phänomen“ synonym verwendet. ${ }^{26}$ Physikalische Effekte sind aber nichts anderes als empirische Regularitäten, also allgemeine Sachverhalte. Als Hall-Effekt bezeichnet man beispielsweise den Sachverhalt, dass in stromdurchflossenen elektrischen Leitern in einem Magnetfeld eine elektrische Spannung auftritt. Wenngleich also nicht alles, was als Phänomen bezeichnet wird, der ontologischen Klasse der Sachverhalte angehört, so doch immerhin eine verhältnismäßig große Zahl Bezugsgegenstände des Ausdrucks.

Philosophische Begriffsanalysen sollten zwar so deskriptiv wie möglich sein, sie dürfen aber auch präskriptive Elemente enthalten. Die hier vorgeschlagene Auffassung von Phänomenen als Sachverhalten ist ein solches präskriptives Element: Sie ist nicht vollständig durch den Sprachgebrauch gedeckt, aber sie passt zu zahlreichen Charakteristika des Sprachgebrauchs und ermöglicht inhaltliche Kürze. So erspart sie mir und dem Leser die ständige Verwendung von Formulierungen wie „Phänomene sind Sachverhalte, Ereignisse, Zustände, Prozesse, Eigenschaften von Gegenständen etc., die durch wissenschaftliche Theorien erklärt werden sollen." Man kann diesen Punkt auch anders formulieren: Worauf es mir, genau wie Bogen und Woodward, an erster Stelle ankommt, sind die funktionalen Rollen von Phänomenen und all diejenigen Entitäten, die diese Rollen innehaben, bezeichne ich als Sachverhalte. Jemandem, der den Schritt, Phänomene als Sachverhalte aufzufassen, nicht mitgehen will, empfehle ich an den Stellen, wo ich von Phänomenen als Sachverhalten spreche, stattdessen immer den Ausdruck „Sachverhalte über Phänomene“ einzusetzen. Hierdurch wird meine weitere Argumentation nicht beeinflusst. Da die Auffassung von Phänomenen als Sachverhalten im zuvor erläuterten Sinne mehr eine pragmatische Entscheidung und keine starke ontologische These ist, ist zu-

${ }^{26}$ Siehe obiges Zitat von Tonomura und Nori (2008). Vgl. auch Falkenburg (2009). 
dem eine weitere Klärung des Sachverhaltsbegriffs an dieser Stelle nicht erforderlich, vielmehr genügen die kurzen Bemerkungen, die zu Beginn dieses Abschnitts gegeben wurden.

Aber hat es nicht dennoch weit reichende Konsequenzen, dass ich Phänomene als Sachverhalte auffasse? Schließlich weckt die Rede von Sachverhalten allerlei philosophische Assoziationen. Man denkt an Theorien, wie sie in Wittgensteins Tractatus oder Armstrongs A World of States of Affairs entwickelt werden. ${ }^{27}$ Lade ich mir mit der Behauptung, Phänomene seien Sachverhalte, nicht unnötigen metaphysischen Ballast auf, weil ich mich auf eine Sachverhaltsontologie und eine Wahrmachertheorie, die behauptet, Tatsachen seien die Wahrmacher von Sätzen, festlege? Auch dies ist meines Erachtens nicht der Fall. Mir persönlich scheint letztere Auffassung plausibel ${ }^{28}$ und erstere unplausibel, ${ }^{29}$ aber dies ist an dieser Stelle nicht entscheidend und wird in meinen Ausführungen keineswegs vorausgesetzt. Jemand, der diese Positionen nicht teilt, kann ohne weiteres seine jeweilige philosophische Theorie unserer alltäglichen und wissenschaftlichen Rede über Tatsachen und darüber, dass eine Aussage wahr ist, weil dieses oder jenes der Fall ist, zugrunde legen und meine weiteren Ausführungen entsprechend interpretieren.

Auf einen weiteren Punkt möchte ich ebenfalls noch hinweisen: Wenn ich davon spreche, dass Phänomene Sachverhalte sind, dann ist dies einigen Philosophen nicht stark genug. Diese behaupten, dass nur Tatsachen als Relata der Erklärungs- und Belegrelationen in Frage kommen - zumindest wenn es um die für Phänomene charakteristischen Relata des Explanandum und des Belegs geht. Diese stärkere Behauptung werde ich hier nicht zugrunde legen, zum einen da ich sie für falsch halte. Mir erscheint es vielmehr, aus Gründen, die ich hier nicht detailliert ausführen kann, plausibel, dass auch ein Wissenschaftlicher Antirealist, der die Aussagen der Quantenphysik nicht für wahr hält, sinnvoll von der Erklärung eines Quantenphänomens sprechen kann. ${ }^{30}$ Zum anderen, und das ist hier der wichtigere Punkt, würde diese Annahme bereits voraussetzen, was im

\footnotetext{
${ }^{27}$ Vgl. Wittgenstein ([1921] 1984) und Armstrong (1997).

${ }^{28}$ Eine entsprechende Ausarbeitung liefert Schantz (1996).

${ }^{29}$ Michael Esfeld führt aus, warum man durch die Annahme, Tatsachen seien Bezugsgegenstände und Wahrmacher von Propositionen, nicht zwingend auf eine Sachverhalts- bzw. Tatsachenontologie festgelegt ist. Vielmehr ist erstere Auffassung durchaus damit verträglich, dass Tatsachen ontologisch auf Gegenstände und ihre Eigenschaften reduziert werden können. Vgl. Esfeld (2004).

${ }^{30}$ Vgl. van Fraassen (1980), S. 151-153 und Hitchcock (1992).
} 
Verlauf dieser Arbeit erst diskutiert werden soll. Erst in Teil B wird der erkenntnistheoretische Status der Phänomene, von denen wissenschaftliche Theorien (vermeintlich) handeln, zur Debatte stehen. Ob das Phänomen des Aharonov-Bohm-Effekts in obigem Zitat eine Tatsache ist oder nicht, ist gerade Gegenstand der Wissenschaftlichen Realismusdebatte, denn den Aharonov-Bohm-Effekt selbst beobachten wir nicht, sondern wir erschließen ihn aus bestimmten experimentell gewonnenen Daten. Wissenschaftliche Realisten gehen davon aus, dass wir auf diese Weise eine allgemeine Tatsache über die Natur entdecken, Antirealisten bestreiten dies. Dennoch bestreiten Antirealisten natürlich nicht, dass der Aharonov-Bohm-Effekt ein Phänomen ist, mit dem sich Wissenschaftler beschäftigen und das sie zu erklären versuchen. Sie sprechen ihm nur einen anderen erkenntnistheoretischen Status zu als beispielsweise der beobachtbaren Tatsache, dass die Blätter von Laubbäumen im Herbst braun werden. Aus diesem Grund fasse ich Phänomene als Sachverhalte und nicht als Tatsachen auf und lasse damit offen, ob wir gute Gründe dafür haben, anzunehmen, dass die entsprechenden Sachverhalte auch tatsächlich bestehen. 
